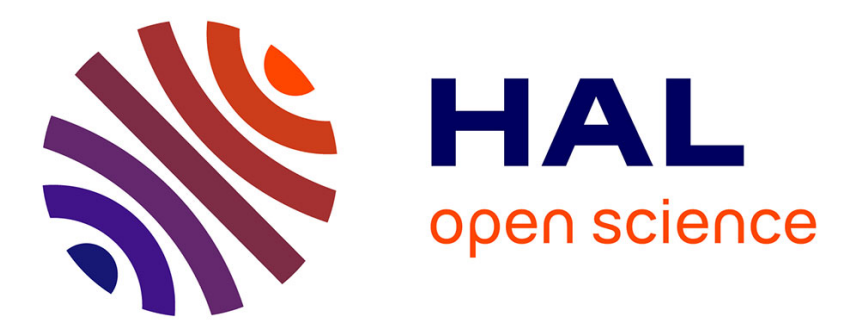

\title{
Convergence of utility functions and convergence of optimal strategies
}

Clotilde Napp, Elyès Jouini

\section{To cite this version:}

Clotilde Napp, Elyès Jouini. Convergence of utility functions and convergence of optimal strategies. Finance and Stochastics, 2004, VIII (1), pp.133-144. halshs-00151579

\section{HAL Id: halshs-00151579 \\ https://shs.hal.science/halshs-00151579}

Submitted on 4 Jun 2007

HAL is a multi-disciplinary open access archive for the deposit and dissemination of scientific research documents, whether they are published or not. The documents may come from teaching and research institutions in France or abroad, or from public or private research centers.
L'archive ouverte pluridisciplinaire HAL, est destinée au dépôt et à la diffusion de documents scientifiques de niveau recherche, publiés ou non, émanant des établissements d'enseignement et de recherche français ou étrangers, des laboratoires publics ou privés. 


\title{
Convergence of utility functions and convergence of optimal strategies*
}

\author{
Elyès JOUINI \\ CEREMADE, Université Paris IX-Dauphine, \\ Place du Maréchal de Lattre de Tassigny, 75116 Paris Cédex and CREST. \\ Clotilde NAPP \\ CEREMADE, Université Paris IX-Dauphine, \\ Place du Maréchal de Lattre de Tassigny, 75116 Paris Cédex and CREST.
}

March 19, 2003

\begin{abstract}
In this paper we study the stability (in the $L^{p}$ as well as for the almost sure convergence sense) of the optimal investment-consumption strategy with respect to the choice of the utility function.
\end{abstract}

\section{Introduction}

In this paper we study in a given quite general complete financial market the stability of the optimal investment-consumption strategy - as defined by Merton (1971) - with respect to the choice of the utility function. More precisely, we consider a sequence of utility functions that converges pointwise and satisfies a given growth condition. For each utility function, we assume that the usual conditions for the existence of an optimal strategy are fulfilled, and we prove the almost sure as well as the $L^{p}$-convergence $(p \geq 1)$ of the optimal wealth and consumption at each date. Moreover, we obtain the $L^{1}$-convergence of the optimal investment process in the general case and its almost sure convergence in a Markov setting if we further assume that the marginal utilities are convex.

Our results can be seen as robustness properties for the optimal strategies and generalize analogous results obtained by Grasselli (2001) in a specific setting (HARA utility functions and interest rates assets in CIR framework). They permit to obtain an approximate optimal strategy when we have an imperfect estimation of the utility function. For instance, the optimal strategy for the exponential (resp. logarithmic) utility function appears as the limit of the optimal strategy for the power utility functions.

\footnotetext{
*The authors want to thank an anonymous referee for his careful reading and useful comments and suggestions (in particular, the development on the speed of convergence).
} 


\section{The model}

\subsection{The financial market}

We fix a finite-time horizon $\mathbb{T} \equiv[0, T]$, on which we are going to treat our problem: $T$ corresponds to the terminal date for all economic activity under consideration. All processes that we shall encounter in this paper are defined on $\mathbb{T}$.

The market model is the same as in Karatzas (1989). We have one bond with price at time $t$ denoted by $S_{t}^{0}$ such that

$$
d S_{t}^{0}=r_{t} S_{t}^{0} d t, \quad S_{0}^{0}=1
$$

and $m$ stocks with price per share at time $t$ denoted by $S_{t}=\left(S_{t}^{1}, \ldots, S_{t}^{m}\right)^{*}$, satisfying the equation

$$
d S_{t}=\operatorname{diag}\left(S_{t}\right)\left[\mu_{t} d t+\sigma_{t} d W_{t}\right], \quad S_{0}=(1, \ldots, 1)^{*} .
$$

Here, $W=\left\{\left(W_{t}^{1}, \ldots, W_{t}^{m}\right)^{*} ; t \in \mathbb{T}\right\}$ is an $m$-dimensional Brownian motion on a probability space $(\Omega, F, P)$ and we let $\left(F_{t}\right)_{t \in \mathbb{T}}$ denote the $P$-augmentation of the natural filtration generated by $W$. We assume that $F_{T}=F$.

The $\mathbb{R}$-valued process $\left\{r_{t} ; t \in \mathbb{T}\right\}$, the $\mathbb{R}^{m}$-valued process $\left\{\mu_{t} ; t \in \mathbb{T}\right\}$ as well as the volatility $(m \times m)$-matrix-valued process $\left\{\sigma_{t} ; t \in \mathbb{T}\right\}$ are the coefficients of the model and are taken to be progressively measurable with respect to $\left(F_{t}\right)_{t \in \mathbb{T}}$ and bounded uniformly in $(t, \omega)$ in $\mathbb{T} \times \Omega$. We assume that for all $t$ in $\mathbb{T}$, the volatility matrix $\sigma_{t}$ is invertible and that the norm of $\sigma_{t}^{-1}$ is uniformly bounded. As usual, the $m$-dimensional relative risk process $\theta=\left\{\theta_{t} ; t \in \mathbb{T}\right\}$ can then be defined by

$$
\theta_{t} \equiv \sigma_{t}^{-1}\left(\mu_{t}-r_{t} \mathbf{1}_{m}\right), \quad t \in \mathbb{T},
$$

where $\mathbf{1}_{m}$ is the vector of $\mathbb{R}^{m}$ whose components are all equal to one and there exists a unique equivalent martingale measure $Q$ for $\left\{S_{t} ; t \in \mathbb{T}\right\}$ given by

$$
\frac{d Q}{d P}=\mathcal{E}_{T}(-\theta) \equiv \exp \left\{\int_{0}^{T}-\left(\theta_{s}\right)^{*} d W_{s}-1 / 2 \int_{0}^{T}\left\|\theta_{s}\right\|^{2} d s\right\} .
$$

In the remainder of the paper, we shall denote by $\left\{M_{t} ; t \in \mathbb{T}\right\}$ the martingale process given by $M_{t}=\mathcal{E}_{t}(-\theta)$ and by $\left\{\tilde{M}_{t} ; t \in \mathbb{T}\right\}$ the discounted process $\tilde{M}=\frac{M}{S^{0}}$.

\subsection{The agent's problem}

Let us now consider an economic agent who invests in the financial market. The agent starts out with an initial capital $x$ and can decide the amounts $\pi_{t}^{i}$ that he invests at time $t$ in the $i$-th stock, and the rate $c_{t}$ at which he withdraws funds for consumption. An investmentconsumption strategy is then defined by an $m$-dimensional process $\pi$ such that $\int_{0}^{T}\left\|\pi_{t}\right\|^{2} d t<\infty$ a.s. and a nonnegative, progressively measurable, real-valued process $c$ satisfying $\int_{0}^{T} c_{t} d t<\infty$ a.s. Assuming that at each time $t$ sales must finance consumption and purchases, the wealth process $X_{t}^{x ; \pi, c}$ associated to an investment-consumption strategy $(\pi, c)$, satisfies the following equation

$$
X_{t}^{x ; \pi, c}=x+\int_{0}^{t}\left(r_{s} X_{s}^{x ; \pi, c}-c_{s}\right) d s+\int_{0}^{t}\left(\pi_{s}\right)^{*} \sigma_{s} d W_{s}^{Q}
$$


where $\left(W_{t}^{Q}\right)_{t \in \mathbb{T}}$ is the $Q$-Brownian motion for $\left(F_{t}\right)_{t \in \mathbb{T}}$, defined by $W_{t}^{Q} \equiv W_{t}+\int_{0}^{t} \theta_{s} d s$ for all $t$ in $\mathbb{T}$.

The agent's preferences are represented by a utility function $U$ for consumption and terminal wealth given by

$$
U(c, X)=E\left[\int_{0}^{T} u\left(t, c_{t}\right) d t+V(X)\right] .
$$

We introduce the following notations. We say that a function $F: R_{+}^{*} \rightarrow R$ satisfies property (P1) if it is of class $C^{1}$, strictly increasing and strictly concave; (P2) if it satisfies Inada's conditions, i.e. $F^{\prime}(0)=\infty$ and $F^{\prime}(\infty)=0 ;\left(\mathbf{P 3}\left(k_{1}, k_{2}, \delta\right)\right)$ if $F(x) \leq k_{1}+k_{2} x^{\delta}$ where $\delta<$ $1, k_{1}>0$ and $k_{2}>0$. For $\left(\delta, k_{1}, k_{2}\right) \in(0,1) \times\left(\mathbb{R}_{+}^{*}\right)^{2}$, we introduce the following condition.

Condition $\left(\mathbf{U}\left(k_{1}, k_{2}, \delta\right)\right): V$ as well as $u(t, \cdot)$, for all $t$, satisfy properties $(\mathbf{P 1}),(\mathbf{P 2}),\left(\mathbf{P 3}\left(k_{1}, k_{2}, \delta\right)\right)$ and $u: \mathbb{T} \times R_{+}^{*} \rightarrow R$ is continuous ${ }^{1}$.

Under $\mathbf{U}\left(k_{1}, k_{2}, \delta\right)$, we shall denote by $u_{c}(t, \cdot)$ the derivative of $u(t, \cdot)$ and by $I_{V}$ and $I_{u}(t, \cdot)$ the inverse functions of $V^{\prime}$ and $u_{c}(t, \cdot)$. These functions are obviously continuous and strictly decreasing.

Since the agent is endowed with the initial capital $x>0$, and there is no exogenous endowment during the trading period $\mathbb{T}$, his problem $(\mathcal{P})$ is then

$$
\sup _{(\pi, c) \in A(x)} U\left(c, X_{T}^{x ; \pi, c}\right)
$$

where we denote by $A(x)$ the set of investment-consumption strategies $(\pi, c)$ such that $X_{t}^{x ; \pi, c} \geq 0$ for all $t \in \mathbb{T}$ and $E\left[\int_{0}^{T} u_{-}\left(t, c_{t}\right) d t+V_{-}\left(X_{T}^{x ; \pi, c}\right)\right]<\infty$.

Let us recall the following classical characterization of the optimal strategy, see e.g. Karatzas (1989).

Proposition 2.1. Under the condition $\left(\mathbf{U}\left(k_{1}, k_{2}, \delta\right)\right)$, there exists a unique optimal investmentconsumption strategy $\left(\pi^{*}, c^{*}\right)$ characterized by the existence of a multiplier $\gamma^{*}>0$ such that

$$
\begin{gathered}
\gamma^{*} \tilde{M}_{t}=u_{c}\left(t, c_{t}^{*}\right) \quad t \in \mathbb{T} \\
\gamma^{*} \tilde{M}_{T}=V^{\prime}\left(X_{T}^{x ; \pi^{*}, c^{*}}\right) \\
E\left[\int_{0}^{T} \tilde{M}_{t} c_{t}^{*} d t+\tilde{M}_{T} X_{T}^{x ; \pi^{*}, c^{*}}\right]=x .
\end{gathered}
$$

\footnotetext{
${ }^{1}$ Notice that property (P3) was introduced for utility functions in Karatzas et al. (1991, condition (5-4)) Note that if $u$ satisfies $(\mathbf{P 1})$ and $(\mathbf{P 2})$ and if $u(\infty)>0$, then property (P3) is weaker than each of the subsequent assertions

1) There is $x_{0}>0, \alpha<1$ and $\beta>1$, such that $U^{\prime}(\beta x)<\alpha U^{\prime}(x)$, for $x>x_{0}$, (Karatzas et al. (1991)),

2) $A E(u)<1$, where $A E(u)=\limsup \frac{x u^{\prime}(x)}{u(x)}$, (Kramkov-Schachermayer (1999)),

3) $x u^{\prime}(x) \leq a+(1-b) u(x)$, for $x>0, a \geq 0,0<b \leq 1$ (Cvitanic-Karatzas (1996)),

4) $u^{\prime \prime}$ exists and $\lim _{x \rightarrow \infty} \frac{\left(u^{\prime}(x)\right)^{\alpha}}{u^{\prime \prime}(x)}=0$ for $\alpha>2$ (Karatzas et al. (1987)).
} 
Let us now consider a sequence of utility functions $\left(u^{n}, V^{n}\right)_{n \in \mathbb{N}}$ converging pointwise to $\left(u^{\infty}, V^{\infty}\right)$.

We make the following growth assumption.

Assumption (A1) : The utility functions $\left(u^{n}, V^{n}\right)_{n \in \mathbb{N} \cup\{\infty\}}$ satisfy condition $\left(\mathbf{U}\left(k_{1}, k_{2}, \delta\right)\right)$ for the same $\left(k_{1}, k_{2}, \delta\right)$.

Let us denote by $\left(\pi^{n}, c^{n}\right)$, (resp. $\left.\left(\pi^{\infty}, c^{\infty}\right)\right)$, the optimal solution of the problem $\mathcal{P}^{n}$ defined by $\left(u^{n}, V^{n}\right)$ (resp. $\mathcal{P}^{\infty}$ defined by $\left.\left(u^{\infty}, V^{\infty}\right)\right)$ and let us denote by $\left\{X_{t}^{n} ; t \in \mathbb{T}\right\}$ and $\left\{X_{t}^{\infty} ; t \in \mathbb{T}\right\}$ the associated wealth processes.

Our aim is to study robustness properties of the optimal consumption and wealth, as well as of the optimal investment strategy with respect to the utility function, and we shall therefore study the convergence of $\left(\pi^{n}, c^{n}, X^{n}\right)_{n \in \mathbb{N}}$.

We shall denote by $\gamma^{n}$ (resp. $\gamma^{\infty}$ ) the multiplier associated with $\mathcal{P}^{n}$ (resp. $\mathcal{P}^{\infty}$ ). The paper is structured as follows. We start by studying the convergence of $I_{u^{n}}$ and $I_{V^{n}}$ (Lemma 2.2), then the convergence of the sequence $\left(\gamma^{n}\right)$ of multipliers (Lemma 2.4). We obtain in Proposition (2.5) convergence results on $\left(c^{n}\right)$ and $\left(X^{n}\right)$ and Proposition (2.6) studies the speed of convergence. Finally, Propositions (2.7), (2.8), (2.9) deal with convergence results on the sequence of optimal portfolio policies $\left(\pi^{n}\right)$.

\section{Lemma 2.2. Under Assumption (A1),}

1. The sequences $\left(I_{u^{n}}\right)$ and $\left(I_{V^{n}}\right)$ converge respectively to $I_{u^{\infty}}$ and $I_{V^{\infty}}$.

2. There exist positive real numbers $K_{1}, K_{2}$ and $\beta$ such that for all $n \in \mathbb{N} \cup\{\infty\}, I_{u^{n}}(t, \cdot)$ for all $t$ and $I_{V^{n}}$ satisfy property $\left(\mathbf{P 3}\left(K_{1}, K_{2},-\beta\right)\right)$.

Proof 1) Let $x \in \mathbb{R}_{+}^{*}, y^{n}=I_{V^{n}}(x)$ and $y=I_{V^{\infty}}(x)$. Let $\varepsilon>0$ be given. By strict monotonicity, we have $\left(V^{\infty}\right)^{\prime}(y-\varepsilon)>x$ and $\left(V^{\infty}\right)^{\prime}(y+\varepsilon)<x$. Since the functions $V^{n}$ are concave, $C^{1}$ and converge pointwise to $V^{\infty}$, it is well-known that $\left(V^{n}\right)^{\prime}$ converges to $\left(V^{\infty}\right)^{\prime}$ and the convergence is uniform on compact subsets of $\mathbb{R}_{+}^{*}$ (see e.g. Rockafellar Th. 25.7, p.248). Therefore, for $n$ sufficiently large, we have $\left(V^{n}\right)^{\prime}(y-\varepsilon)>x$ and $\left(V^{n}\right)^{\prime}(y+\varepsilon)<x$ and consequently $y^{n} \in$ $(y-\varepsilon, y+\varepsilon)$.

2) By concavity, we have $\left(V^{n}\right)^{\prime}(y) \leq \frac{\left(V^{n}\right)(y)-V^{n}(1)}{y-1}$ for $y \geq 1$, then $\left(V^{n}\right)^{\prime}(y) \leq \frac{k_{2} y^{\delta}}{y-1}+$ $\frac{k_{1}-V^{n}(1)}{y-1}$. Since $V^{n}(1)$ converges to $V^{\infty}(1)$, this sequence is bounded, and there exist positive constants $k_{3}$ and $k_{4}$, that do not depend upon $n$, such that for $y \geq 2,\left(V^{n}\right)^{\prime}(y) \leq \frac{k_{2} y^{\delta}}{y-1}+\frac{k_{3}}{y-1} \leq$ $k_{4} y^{\delta-1}$. This leads to $I_{V^{n}}(y) \leq\left(\frac{y}{k_{4}}\right)^{\frac{1}{\delta-1}}$ for $\left.\left.y \in\right] 0, k_{5}\right]$ where $k_{5}=2^{\delta-1} k_{4}$. Since $I_{V^{n}}$ is nonincreasing, we have $I_{V^{n}}(y) \leq I_{V^{n}}\left(k_{5}\right)$ for $y \geq k_{5}$ and the convergence of $I_{V^{n}}\left(k_{5}\right)$ to $I_{V^{\infty}}\left(k_{5}\right)$ gives us the conclusion.

We shall need the next technical lemma.

Lemma 2.3. For any real number $l, \sup _{t \in \mathbb{T}} E\left[\tilde{M}_{t}^{l}\right]<\infty$.

Proof This result is standard in our "Karatzas-like" framework.

Lemma 2.4. Under Assumption (A1), the sequence $\left(\gamma^{n}\right)$ converges to $\gamma^{\infty}$. 
Proof For all $y \in \mathbb{R}_{+}^{*}$, we introduce

$$
\varphi^{n}(y) \equiv E\left[\int_{0}^{T} \tilde{M}_{t} I_{u^{n}}\left(t, y \tilde{M}_{t}\right) d t+\tilde{M}_{T} I_{V^{n}}\left(y \tilde{M}_{T}\right)\right] .
$$

By Lemma 2.2, we have for some positive constants $K_{1}, K_{2}, \beta$,

$$
E\left[\int_{0}^{T} \tilde{M}_{t} I_{u^{n}}\left(t, y \tilde{M}_{t}\right) d t\right] \leq E\left[\int_{0}^{T}\left(K_{1} \tilde{M}_{t}+K_{2} y^{-\beta} \tilde{M}_{t}^{1-\beta}\right) d t\right]
$$

which by Fubini's Theorem and Lemma 2.3, is finite. The same argument on $V^{n}$ leads to $\varphi^{n}(y)<\infty$ for all $y \in \mathbb{R}_{+}^{*}$. The continuity of $I_{V^{n}}$ and of $I_{u^{n}}(t, \cdot)$ for all $n$, and Lebesgue Theorem give us the continuity of the real-valued function $\varphi^{n}$ for all $n$.

The same dominated convergence argument gives us the convergence of $\varphi^{n}$ to $\varphi^{\infty}$. All these functions are continuous and (strictly) decreasing, consequently $\left(\varphi^{n}\right)^{-1}$ converges to $\left(\varphi^{\infty}\right)^{-1}$ as in the proof of Lemma 2.2 and $\gamma^{n}=\left(\varphi^{n}\right)^{-1}(x)$ converges to $\gamma^{\infty}=\left(\varphi^{\infty}\right)^{-1}(x)$.

Proposition 2.5. Under Assumption (A1), the sequences $\left(X_{t}^{n}\right)$ and $\left(c_{t}^{n}\right)$ converge almost surely and in $L^{p}-$ norm (for $\left.1 \leq p<\infty\right)$ respectively to $\left(X_{t}^{\infty}\right)$ and $\left(c_{t}^{\infty}\right)$.

Proof We have $X_{T}^{n}=I_{V^{n}}\left(\gamma^{n} \tilde{M}_{T}\right)$ and $\gamma^{n}$ converges to $\gamma^{\infty}>0$ (Proposition 2.4). For all $\omega \in \Omega$, the sequence $\left(\gamma^{n} \tilde{M}_{T}(\omega)\right)_{n}$ belongs to a compact subset of $\mathbb{R}_{+}^{*}$. Furthermore, the sequence $I_{V^{n}}$ is a sequence of decreasing functions converging pointwise to $I_{V^{\infty}}$ (Lemma 2.2). We have then the uniform convergence on any compact subset of $\mathbb{R}_{+}^{*}$ (by Dini's Theorem) which gives us the almost sure convergence of $X_{T}^{n}$ to $X_{T}^{\infty}$. Analogously, we obtain the almost sure convergence of $c_{t}^{n}$ to $c_{t}^{\infty}$.

For $t \in \mathbb{T}, X_{t}^{n}=\frac{1}{\tilde{M}_{t}} E_{t}\left[\tilde{M}_{T} X_{T}^{n}+\int_{t}^{T} \tilde{M}_{s} c_{s}^{n} d s\right]$. The random variable $\tilde{M}_{T} X_{T}^{n}$ converges almost surely to $\tilde{M}_{T} X_{T}^{\infty}$ and is dominated by $K_{1} \tilde{M}_{T}+K_{2} \bar{\gamma}^{-\beta} \tilde{M}_{T}^{1-\beta}$ where $\bar{\gamma}$ is a positive lower bound for the sequence $\left(\gamma^{n}\right)$. We have then the almost sure convergence of $\frac{1}{\bar{M}_{t}} E_{t}\left[\tilde{M}_{T} X_{T}^{n}\right]$ to $\frac{1}{\tilde{M}_{t}} E_{t}\left[\tilde{M}_{T} X_{T}^{\infty}\right]$. Besides, the random variable $\tilde{M} c^{n}$ converges almost surely to $\tilde{M} c^{\infty}$ and is dominated by $K_{1} \tilde{M}+K_{2} \bar{\gamma}^{-\beta} \tilde{M}^{1-\beta}$ which is $\operatorname{in}^{2} L^{1}\left(\Omega \times \mathbb{T}, F \otimes \mathcal{B}_{\mathbb{T}}, P \otimes \lambda_{\mathbb{T}}\right)$ by Fubini's theorem and Lemma 2.3. We have then the almost sure convergence of $X_{t}^{n}$ to $X_{t}^{\infty}$.

Let us show that $\left(X_{T}^{n}\right)$ is dominated by a random variable in $L^{p}$ for some $p \geq 1$. We have $\left|X_{T}^{n}\right|=I_{V^{n}}\left(\gamma^{n} \tilde{M}_{T}\right) \leq K_{1}+K_{2}\left(\bar{\gamma} \tilde{M}_{T}\right)^{-\beta}$. We then obtain the $L^{p}$-convergence of $\left(X_{T}^{n}\right)$ to $X_{T}^{\infty}$ and analogously the $L^{p}$-convergence of $c_{t}^{n}$ to $c_{t}^{\infty}$.

It only remains to prove the $L^{p}$-convergence of $X_{t}^{n}$ to $X_{t}^{\infty}$. We have

$$
\begin{aligned}
\left|X_{t}^{n}\right| \leq & \left|E_{t}\left[\frac{\tilde{M}_{T}}{\tilde{M}_{t}}\left(K_{1}+K_{2}\left(\bar{\gamma} \tilde{M}_{T}\right)^{-\beta}\right)\right]\right| \\
& +\left|E_{t}\left[\int_{t}^{T} \frac{\tilde{M}_{s}}{\tilde{M}_{t}}\left(K_{1}+K_{2}\left(\bar{\gamma} \tilde{M}_{s}\right)^{-\beta}\right) d s\right]\right|
\end{aligned}
$$

\footnotetext{
${ }^{2}$ Where as usual, $\lambda_{\mathbb{T}}$ denotes Lebesgue's measure restricted to the Borel sigma-field $\mathcal{B}_{\mathbb{T}}$.
} 
Let us prove that $E_{t}\left[\int_{t}^{T} \frac{\tilde{M}_{s}}{\tilde{M}_{t}} K_{2}\left(\bar{\gamma} \tilde{M}_{s}\right)^{-\beta} d s\right]$ is in $L^{p}$. The other terms can be similarly handled. We have for $p \geq 1$,

$$
\begin{aligned}
E\left(\left[E_{t}\left[\int_{t}^{T} \frac{\tilde{M}_{s}}{\tilde{M}_{t}} K_{2}\left(\bar{\gamma} \tilde{M}_{s}\right)^{-\beta} d s\right]\right]^{p}\right) & \leq E\left(E_{t}\left[\int_{t}^{T}\left(\frac{\tilde{M}_{s}}{\tilde{M}_{t}} K_{2}\left(\bar{\gamma}_{s}\right)^{-\beta}\right)^{p} d s\right]\right) \\
& \leq K_{2}^{p} \bar{\gamma}^{-\beta p} \int_{t}^{T} E\left(\frac{\tilde{M}_{s}^{(1-\beta) p}}{\tilde{M}_{t}^{p}}\right) d s \\
& \leq K_{2}^{p} \bar{\gamma}^{-\beta p} E\left(\tilde{M}_{t}^{-2 p}\right)^{1 / 2} \int_{t}^{T} E\left(\tilde{M}_{s}^{2(1-\beta) p}\right)^{1 / 2} d s \\
& \leq T K_{2}^{p} \bar{\gamma}^{-\beta p} E\left(\tilde{M}_{t}^{-2 p}\right)^{1 / 2} \sup _{s \in \mathbb{T}} E\left(\tilde{M}_{s}^{2(1-\beta) p}\right)^{1 / 2},
\end{aligned}
$$

which ends the proof.

The next proposition gives an error estimate for the previous convergence. We introduce the following

Assumption (A2) : For all $n \in \mathbb{N} \cup\{\infty\}$, the utility functions $V^{n}$ as well as $u^{n}(t, \cdot)$, for all $t$, are of class $C^{3}$ and have a convex derivative.

Proposition 2.6. Under Assumptions $(A 1)$ and $(A 2)$, if there exist continuous functions $H(t, \cdot)$ and $K: \mathbb{R} \rightarrow \mathbb{R}$ and two positive scalars $\lambda_{1}$ and $\lambda_{2}$ for which, for all $(t, z, n) \in \mathbb{T} \times \mathbb{R} \times \mathbb{N}$

$$
\begin{aligned}
& \frac{\lambda_{1}}{n} H(t, z) \leq\left|I_{u^{n}}(t, z)-I_{u^{\infty}}(t, z)\right| \leq \frac{\lambda_{2}}{n} H(t, z) \\
& \frac{\lambda_{1}}{n} K(z) \leq\left|I_{V^{n}}(z)-I_{V^{\infty}}(z)\right| \leq \frac{\lambda_{2}}{n} K(z)
\end{aligned}
$$

then there exist random variables $L$ and $\left(N_{t}\right)_{t \in \mathbb{T}}$ such that for all $(t, n) \in \mathbb{T} \times \mathbb{N}$ we have

$$
\begin{aligned}
& \left|X_{T}^{n}-X_{T}^{\infty}\right| \leq \frac{1}{n} L \\
& \left|c_{t}^{n}-c_{t}^{\infty}\right| \leq \frac{1}{n} N_{t} .
\end{aligned}
$$

Proof. Notice first that under Assumption $(A 2)$ the function $\varphi^{\infty}$ admits a derivative $\left(\varphi^{\infty}\right)^{\prime}$. Indeed, it is easy to see that since $I_{V^{\infty}}$ is convex under Assumption $(A 2)$, we have $I_{V^{\infty}}^{\prime}(x) \geq$ $\frac{I_{V \infty}(x)-I_{V \infty}(x / 2)}{x / 2} \geq-\frac{K_{1}+K_{2}(x / 2)^{-\beta}}{x / 2}$ so that there exist positive constants $K_{1}^{\prime}$ and $K_{2}^{\prime}$ for which

$$
\left|I_{V \infty}^{\prime}(x)\right| \leq K_{1}^{\prime} x^{-1}+K_{2}^{\prime} x^{-\beta-1}
$$

An analogous inequality can be similarly obtained for $I_{u^{\infty}}^{\prime}(t, \cdot)$, and the differentiability of $\varphi^{\infty}$ stems then from Lebesgue's Theorem and Lemma (2.3). More precisely, for all $y \in \mathbb{R}_{+}^{*}$, we have

$$
\left(\varphi^{n}\right)^{\prime}(y)=E\left[\int_{0}^{T} \tilde{M}_{t}^{2} I_{u^{n}}^{\prime}\left(t, y \tilde{M}_{t}\right) d t+\tilde{M}_{T}^{2} I_{V^{n}}^{\prime}\left(y \tilde{M}_{T}\right)\right],
$$


which does not vanish under Assumption $(A 2)$.

Let us estimate $\left|\gamma^{n}-\gamma^{\infty}\right|$. We have

$$
\begin{aligned}
\left|\varphi^{n}(y)-\varphi^{\infty}(y)\right|= & \mid E\left[\int_{0}^{T} \tilde{M}_{t}\left(I_{u^{n}}\left(t, y \tilde{M}_{t}\right)-I_{u^{\infty}}\left(t, y \tilde{M}_{t}\right)\right) d t\right. \\
& \left.+\tilde{M}_{T}\left(I_{V^{n}}\left(y \tilde{M}_{T}\right)-I_{V^{\infty}}\left(y \tilde{M}_{T}\right)\right)\right] \mid \\
\leq & \frac{\lambda_{2}}{n} E\left[\int_{0}^{T} \tilde{M}_{t} H\left(t, y \tilde{M}_{t}\right) d t+\tilde{M}_{T} K\left(y \tilde{M}_{T}\right)\right] .
\end{aligned}
$$

It suffices to remark that $\tilde{M}_{T} K\left(y \tilde{M}_{T}\right) \leq n \frac{1}{\lambda_{1}} \tilde{M}_{T}\left|I_{V^{n}}\left(y \tilde{M}_{T}\right)-I_{V^{\infty}}\left(y \tilde{M}_{T}\right)\right|$ and that $\tilde{M}_{t} H\left(t, y \tilde{M}_{t}\right)$ can be bounded from above similarly to obtain through Lemmas (2.2) and (2.3) the integrability of the right term which we shall denote by $\frac{1}{n} J(y)$.

Let us now prove the existence of a constant $B$ such that $\left|\gamma^{n}-\gamma^{\infty}\right| \leq \frac{B}{n}$ for all $n$. Suppose that this assertion is not true; this means that for all positive constant $B$, there exists $n>B$ such that $\left|\gamma^{n}-\gamma^{\infty}\right|>\frac{B}{n}$.

We know that $\left|x-\varphi^{\infty}\left(\left(\varphi^{n}\right)^{-1}(x)\right)\right| \leq \frac{1}{n} J\left(\left(\varphi^{n}\right)^{-1}(x)\right)$ and then

$$
\left|\left(\varphi^{\infty}\right)^{\prime}\left(x_{n}\right)\right|=\frac{\left|x-\varphi^{\infty}\left(\left(\varphi^{n}\right)^{-1}(x)\right)\right|}{\left|\left(\varphi^{\infty}\right)^{-1}(x)-\left(\varphi^{n}\right)^{-1}(x)\right|}<\frac{J\left(\left(\varphi^{n}\right)^{-1}(x)\right)}{B}
$$

where $x_{n}$ is a given element between $\left(\varphi^{n}\right)^{-1}(x)$ and $\left(\varphi^{\infty}\right)^{-1}(x)$. Let $B$ go to infinity, then $\left(\varphi^{n}\right)^{-1}(x)$ and $x_{n}$ go to $\left(\varphi^{\infty}\right)^{-1}(x)$ and $\left(\varphi^{\infty}\right)^{\prime}\left(\left(\varphi^{\infty}\right)^{-1}(x)\right)$ is then equal to zero which contradicts Assumption $(A 2)$.

We have

$$
\begin{aligned}
\left|X_{T}^{n}-X_{T}^{\infty}\right|= & \left|I_{V^{n}}\left(\gamma^{n} \tilde{M}_{T}\right)-I_{V^{\infty}}\left(\gamma^{\infty} \tilde{M}_{T}\right)\right| \\
& \leq J_{n}^{1}+J_{n}^{2} \\
\leq & \frac{\lambda_{2}}{n} K\left(\gamma^{n} \tilde{M}_{T}\right)+\left|I_{V^{\infty}}^{\prime}\left(C_{n}\right)\right|\left|\gamma^{n}-\gamma^{\infty}\right| \tilde{M}_{T} \\
\leq & \frac{\lambda_{2}}{n} K\left(\gamma_{n} \tilde{M}_{T}\right)+\left|I_{V_{\infty}}^{\prime}\left(C_{n}\right)\right| \frac{1}{n} B \tilde{M}_{T} \\
\leq & \frac{1}{n} H
\end{aligned}
$$

for $J_{n}^{1} \equiv\left|I_{V^{n}}\left(\gamma^{n} \tilde{M}_{T}\right)-I_{V^{\infty}}\left(\gamma^{n} \tilde{M}_{T}\right)\right|$ and $J_{n}^{2} \equiv\left|I_{V^{\infty}}\left(\gamma^{n} \tilde{M}_{T}\right)-I_{V^{\infty}}\left(\gamma^{\infty} \tilde{M}_{T}\right)\right|$. Now, by assumption, $J_{n}^{1} \leq \frac{\lambda_{2}}{n} K\left(\gamma^{n} \tilde{M}_{T}\right) \leq \frac{1}{n} \bar{K}$, where $\bar{K}$ is the a.s. finite random variable given by $\bar{K} \equiv \sup _{n} K\left(\gamma^{n} \tilde{M}_{T}\right)$. On the other hand, if we introduce for all $n$ such that $\gamma^{n} \neq \gamma^{\infty}$ the random variable $C_{n} \equiv \frac{I_{V \infty}\left(\gamma^{n} \tilde{M}_{T}\right)-I_{V}\left(\gamma^{\infty} \tilde{M}_{T}\right)}{\left(\gamma^{n}-\gamma^{\infty}\right) M_{T}}$, then $J_{n}^{2}=\left|C_{n}\right|\left|\gamma^{n}-\gamma^{\infty}\right| \tilde{M}_{T} \leq\left|C_{n}\right| \frac{1}{n} B \tilde{M}_{T} \leq$ 


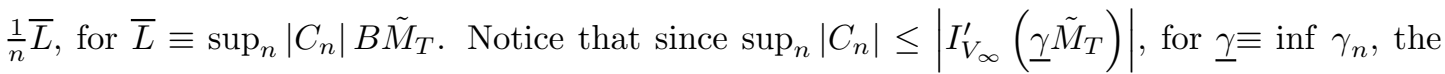
random variable $\bar{L}$ is a.s. finite.

The sequence $\left(c_{n}\right)$ is treated analogously.

We now consider robustness properties of the optimal portfolio policy, or equivalently convergence issues for the sequence of processes $\left(\pi^{n}\right)$.

Proposition 2.7. The sequence $\left(\pi^{n}\right)$ converges to $\pi$ in $L^{1}\left(\Omega \times \mathbb{T}, F \otimes \mathcal{B}_{\mathbb{T}}, P \otimes \lambda_{\mathbb{T}}\right)$

Proof First remark that $\left\|\sigma^{-1}\right\|$ is uniformly bounded. Since

$$
d\left(\frac{X_{t}^{x ; \pi, c}}{S_{t}^{0}}\right)=-\left(\frac{c_{s}}{S_{s}^{0}}\right) d s+\frac{1}{S_{s}^{0}}\left(\pi_{s}\right)^{*} \sigma_{s} d W_{s}^{Q}
$$

we get by Ito's isometry, that

$$
E^{Q}\left[\int_{0}^{T}\left\{\frac{\left(\pi_{s}^{n}-\pi_{s}^{\infty}\right) \sigma_{s}}{S_{s}^{0}}\right\}^{2} d s\right]=E^{Q}\left[\left(\frac{X_{T}^{n}-X_{T}^{\infty}}{S_{T}^{0}}+\int_{0}^{T} \frac{c_{s}^{n}-c_{s}^{\infty}}{S_{s}^{0}} d s\right)^{2}\right]
$$

We know that $X_{T}^{n}$ converges to $X_{T}^{\infty}$ in $L^{2}(Q)$ (see Proposition 2.5 and Lemma 2.3). Besides $\int_{0}^{T} c_{s}^{n} d s$ converges to $\int_{0}^{T} c_{s}^{\infty} d s$ in $L^{2}(Q)$ or equivalently $E^{Q}\left[\left(\int_{0}^{T}\left(c_{s}^{n}-c_{s}^{\infty}\right) d s\right)^{2}\right] \rightarrow 0$. This stems from Jensen's inequality and the fact that the random variable $c^{n}$ converges almost surely to $c^{\infty}$ and is dominated by $K_{1}+K_{2} \bar{\gamma}^{-\beta} \tilde{M}^{-\beta}$ which is in $L^{2}\left(\Omega \times \mathbb{T}, F \otimes \mathcal{B}_{\mathbb{T}}, Q \otimes \lambda_{\mathbb{T}}\right)$ by Fubini's Theorem and Lemma 2.3. We have then the convergence of $\left(\pi^{n}\right)$ to $\pi$ in $L^{2}\left(\Omega \times \mathbb{T}, F \otimes \mathcal{B}_{\mathbb{T}}, Q \otimes \lambda_{\mathbb{T}}\right)$. Hölder's inequality concludes the proof.

In order to obtain almost sure convergence results on the optimal portfolios, we shall consider two specific cases, the model with deterministic coefficients and the Markovian model. We introduce the following

Assumption (A2) : The utility functions $V^{n}$ as well as $u^{n}(t, \cdot)$, for all $t$, are of class $C^{3}$ and have a convex derivative.

Proposition 2.8. When the coefficients $r, \mu$ and $\sigma$ are deterministic, and under (A1) and (A2), the sequence $\left(\pi_{t}^{n}\right)$ converges to $\pi_{t}^{\infty}$ almost surely, for all $t$.

Proof We know that in this setting (Karatzas (1989)), the optimal investment strategy is given by

$$
\pi_{t}^{n}=-\left.\left(\sigma_{t}\left(\sigma_{t}\right)^{*}\right)^{-1}\left(\mu_{t}-r_{t} \mathbf{1}_{m}\right) \gamma^{n} \tilde{M}_{t} \frac{\partial}{\partial y}\right|_{\gamma_{n}} E_{t}\left[\int_{t}^{T} \frac{\tilde{M}_{s}}{\tilde{M}_{t}} I_{u^{n}}\left(s, y \frac{\tilde{M}_{s}}{\tilde{M}_{t}}\right) d s+\frac{\tilde{M}_{T}}{\tilde{M}_{t}} I_{V^{n}}\left(y \frac{\tilde{M}_{T}}{\tilde{M}_{t}}\right)\right] .
$$

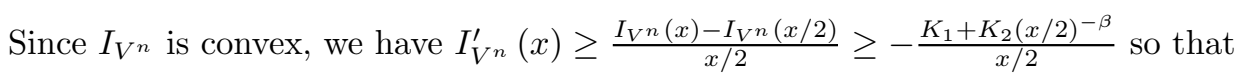

$$
\left|I_{V^{n}}^{\prime}(x)\right| \leq K_{1}^{\prime} x^{-1}+K_{2}^{\prime} x^{-\beta-1}
$$


and then from Lemma 2.3

$$
\pi_{t}^{n}=-\left(\sigma_{t}\left(\sigma_{t}\right)^{*}\right)^{-1}\left(\mu_{t}-r_{t} \mathbf{1}_{m}\right) \gamma^{n} \tilde{M}_{t}^{-1} E_{t}\left[\int_{t}^{T} \tilde{M}_{s}^{2} I_{u^{n}}^{\prime}\left(s, \gamma^{n} \frac{\tilde{M}_{s}}{\tilde{M}_{t}}\right) d s+\tilde{M}_{T}^{2} I_{V^{n}}^{\prime}\left(\gamma^{n} \frac{\tilde{M}_{T}}{\tilde{M}_{t}}\right)\right] .
$$

Since the functions $I_{V^{n}}$ are convex, $C^{1}$ and converge pointwise to $I_{V^{\infty}}$, we have as previously the uniform convergence of $\left(I_{V^{n}}\right)^{\prime}$ to $\left(I_{V^{\infty}}\right)^{\prime}$ on compact subsets of $\mathbb{R}_{+}^{*}$, hence the almost sure convergence of $\tilde{M}_{s}^{2} I_{u^{n}}^{\prime}\left(s, \gamma^{n} \frac{\tilde{M}_{s}}{\tilde{M}_{t}}\right)$ and $\tilde{M}_{T}^{2} I_{V^{n}}^{\prime}\left(\gamma^{n} \frac{\tilde{M}_{T}}{\tilde{M}_{t}}\right)$ to $\tilde{M}_{s}^{2} I_{u^{\infty}}^{\prime}\left(t, \gamma^{\infty} \frac{\tilde{M}_{s}}{\tilde{M}_{t}}\right)$ and $\tilde{M}_{T}^{2} I_{V^{\infty}}^{\prime}\left(\gamma^{\infty} \frac{\tilde{M}_{T}}{\tilde{M}_{t}}\right)$ respectively. Now, Lebesgue's Theorem and Inequation 2.6 give us the almost sure convergence of $\pi_{t}^{n}$ to $\pi_{t}^{\infty}$.

We now consider a Markovian model, i.e., the coefficients $r_{t}, \mu_{t}$ and $\sigma_{t}$ can be written in the form $r\left(t, S_{t}\right), \mu\left(t, S_{t}\right)$ and $\sigma\left(t, S_{t}\right)$ for functions $r: \mathbb{R}_{+} \times \mathbb{R}^{m} \rightarrow \mathbb{R}, \mu: \mathbb{R}_{+} \times \mathbb{R}^{m} \rightarrow \mathbb{R}^{m}$ and $\sigma: \mathbb{R}_{+} \times \mathbb{R}^{m} \rightarrow \mathbb{R}^{m^{2}}$.

Let us introduce as in Cox and Huang (1989) the functions $F^{n}$ defined by

$$
F^{n}\left(t, Z_{t}^{n}, S_{t}\right) \equiv Z_{t}^{n} E\left[\int_{t}^{T}\left(Z_{s}^{n}\right)^{-1} I_{u^{n}}\left(s,\left(Z_{s}^{n}\right)^{-1}\right) d s+\left(Z_{T}^{n}\right)^{-1} I_{V^{n}}\left(\left(Z_{T}^{n}\right)^{-1}\right) \mid\left(Z_{t}^{n}, S_{t}\right)\right]
$$

where $Z^{n} \equiv \frac{1}{\gamma_{n} \tilde{M}}$ is the process defined by

$$
d Z_{t}^{n}=\left(r_{t}+\left\|\theta_{t}\right\|^{2} Z_{t}^{n}\right) d t-\theta_{t} Z_{t}^{n} d W_{t}, Z_{0}^{n}=\frac{1}{\gamma_{n} \tilde{M}_{0}}
$$

When the function $F^{n}$ is twice continuously differentiable, then we know by Cox and Huang (1989, Theorem 2.1) that the optimal investment strategy is given by

$$
\pi_{t}^{n}=\operatorname{diag} S_{t} F_{s}^{n}\left(t, Z_{t}^{n}, S_{t}\right)+\left(\sigma_{t} \sigma_{t}^{*}\right)^{-1}\left[\mu_{t}-r_{t} \mathbf{1}_{m}\right] Z_{t}^{n} F_{z}^{n}\left(t, Z_{t}^{n}, S_{t}\right) .
$$

In order to compute $F_{s}^{n}$ and $F_{z}^{n}$, we introduce the function $f$ given by $f(y)=-1 / y$ for $y \leq-1$ and $f(y)=y^{2}+3 y+3$ for $y \geq-1$, the process $Y^{n}=f^{-1}\left(Z^{n}\right)$ and the following auxiliary function

$$
G^{n}\left(t, Y_{t}^{n}, S_{t}\right) \equiv f\left(Y_{t}^{n}\right) E\left[\int_{t}^{T} \frac{I_{u^{n}}\left(s,\left\{f\left(Y_{s}^{n}\right)\right\}^{-1}\right)}{f\left(Y_{s}^{n}\right)} d s+\frac{I_{V^{n}}\left(\left\{f\left(Y_{T}^{n}\right)\right\}^{-1}\right)}{f\left(Y_{T}^{n}\right)} \mid\left(Y_{t}^{n}, S_{t}\right)\right]
$$

In the next $f^{-1}$ will be denoted by $g$ and we have $g(z)=-1 / z$ for $0<z \leq 1$ and $g(z)=$ $\frac{-3+\sqrt{4 z-3}}{2}$ for $z \geq 1$. Note that $f$ and $g$ are $C^{2}$ and the process $\left(Y_{t}^{n}\right)$ satisfies the following stochastic differential equation

$$
\begin{aligned}
d Y_{t}^{n}= & {\left[g^{\prime}\left(f\left(Y_{t}^{n}\right)\right) f\left(Y_{t}^{n}\right)\left(\left\|\theta_{t}\right\|^{2}+r_{t}\right)+\frac{1}{2} g^{\prime \prime}\left(f\left(Y_{t}^{n}\right)\right)\left\|\theta_{t}\right\|^{2} f\left(Y_{t}^{n}\right)^{2}\right] d t } \\
& -g^{\prime}\left(f\left(Y_{t}^{n}\right)\right) f\left(Y_{t}^{n}\right) \theta_{t} d W_{t} .
\end{aligned}
$$

Since $F^{n}(t,(z, s))=G^{n}(t,(g(z), s))$, the first and second order derivatives of $F^{n}(t,(z, s))$ can be directly obtained from the derivatives of $G^{n}(t,(g(z), s))$ with $F_{s}^{n}(t,(z, s))=G_{s}^{n}(t,(g(z), s))$ and $F_{z}^{n}(t,(z, s))=G_{z}^{n}(t,(g(z), s)) g^{\prime}(z)$. 
It is easy to prove that ${ }_{s} h_{u^{n}}: y \rightarrow\{f(y)\}^{-1} I_{u^{n}}\left(s,\{f(y)\}^{-1}\right)$ and $h_{V^{n}}: y \rightarrow\{f(y)\}^{-1} I_{V^{n}}\left(\{f(y)\}^{-1}\right)$ as well as their first derivatives have a polynomial growth. If we further assume that, for all $n$, there exist positive real valued functions defined on $\mathbb{T}, a_{n}, b_{n}, \alpha_{n}$ and $\beta_{n}$ such that, for all $x>0$, we have

$$
\text { (A3) }\left\{\begin{array}{l}
I_{u^{n}}^{\prime \prime}(t, x) \leq \frac{a_{n}(t)}{x^{\alpha_{n}(t)}}+b_{n}(t) x^{\beta_{n}(t)}, \quad t \in \mathbb{T} \\
I_{V^{n}}^{\prime \prime}(x) \leq \frac{a_{n}(T)}{x^{\alpha}(T)}+b_{n}(T) x^{\beta_{n}(T)}
\end{array}\right.
$$

then the polynomial growth property is also inherited by the second derivatives of ${ }_{s} h_{u^{n}}$ and $h_{V^{n}}$.

Let us now write $2.1,2.2$ and 2.7 compactly as follows

$$
d X_{t}=\mu_{X}\left(t, X_{t}\right) d t+\sigma_{X}\left(t, X_{t}\right) d W_{t}
$$

where $X_{t}=\left(S_{t}^{0}, S_{t}, Y_{t}\right)$ and let us assume

$$
\left\{\begin{array}{l}
\left|\mu_{X}(t, x)\right|^{2}+\left|\sigma_{X}(t, x)\right|^{2} \leq L\left(1+|x|^{2}\right) \\
\forall R, \exists C_{R}, \forall|x|,\left|x^{\prime}\right| \leq R,\left|\mu_{X}(t, x)-\mu_{X}\left(t, x^{\prime}\right)\right|^{2}+\left|\sigma_{X}(t, x)-\sigma_{X}\left(t, x^{\prime}\right)\right|^{2} \leq C_{R}\left|x-x^{\prime}\right|^{2} \\
\left|\mu_{X}(t+h, x)-\mu_{X}(t, x)\right|^{2}+\left|\sigma_{X}(t+h, x)-\sigma_{X}(t, x)\right| \leq L\left(1+|x|^{2}\right) k(h), k \geq 0, k \underset{h \rightarrow 0}{\rightarrow} 0 .
\end{array}\right.
$$

Note that (A4) is weaker than the following:

$\left(\mathbf{A}^{\prime} \mathbf{4}\right)$ : the functions $r(t, s), \mu(t, s)$ and $\sigma(t, s)$ are continuously differentiable with respect to $s$ and the families of functions $\{r(\cdot, s)\}_{s},\{\mu(\cdot, s)\}_{s}$ and $\{\sigma(\cdot, s)\}_{s}$ are uniformly equicontinuous.

Following Gihman and Skorohod, (1972, Lemma 2, p293) the function $G^{n}$ is then differentiable and its differential can be computed by differentiating under the integral sign.

Simple computations give then

$$
\begin{aligned}
F_{z}^{n}\left(t, Z_{t}^{n}, S_{t}\right)= & -E\left[\int_{t}^{T} \gamma_{n}^{2} \tilde{M}_{s}^{2} I_{u^{n}}^{\prime}\left(s, \gamma_{n} \tilde{M}_{s}\right) d s+I_{V^{n}}^{\prime}\left(\gamma_{n} \tilde{M}_{T}\right) \gamma_{n}^{2} \tilde{M}_{T}^{2} \mid\left(Z_{t}^{n}, S_{t}\right)\right] \\
F_{s}^{n}\left(t, Z_{t}^{n}, S_{t}\right)= & -\frac{1}{\tilde{M}_{t}} E\left[\int_{t}^{T} \frac{\partial \tilde{M}_{s}}{\partial S_{t}}\left[\gamma_{n} \tilde{M}_{s} I_{u^{n}}^{\prime}\left(t, \gamma_{n} \tilde{M}_{s}\right)+I_{u^{n}}\left(t, \gamma_{n} \tilde{M}_{s}\right)\right] d s\right. \\
& \left.+\frac{\partial \tilde{M}_{T}}{\partial S_{t}}\left[\gamma_{n} \tilde{M}_{T} I_{V^{n}}^{\prime}\left(\gamma_{n} \tilde{M}_{T}\right)+I_{V^{n}}\left(\gamma_{n} \tilde{M}_{T}\right)\right] \mid\left(\tilde{M}_{t}, S_{t}\right)\right]
\end{aligned}
$$

When $F^{n}$ is twice continuously differentiable, it suffices to prove the a.s. convergence of $F_{s}^{n}\left(t, Z_{t}^{n}, S_{t}\right)$ and $F_{z}^{n}\left(t, Z_{t}^{n}, S_{t}\right)$ to obtain the a.s. convergence of $\pi_{t}^{n}$. As in the proof of Proposition 2.7, we get the convergence of $F_{z}^{n}$. For the convergence of $F_{s}^{n}$, we use as previously the almost sure convergence of $\frac{\partial \tilde{M}_{s}}{\partial S_{t}}\left[\gamma_{n} \tilde{M}_{s} I_{u^{n}}^{\prime}\left(t, \gamma_{n} \tilde{M}_{s}\right)+I_{u^{n}}\left(t, \gamma_{n} \tilde{M}_{s}\right)\right]$ and $\frac{\partial \tilde{M}_{T}}{\partial S_{t}}\left[\gamma_{n} \tilde{M}_{T} I_{V^{n}}^{\prime}\left(\gamma_{n} \tilde{M}_{T}\right)+I_{V^{n}}\left(\gamma_{n} \tilde{M}\right.\right.$ Lebesgue dominated convergence Theorem and the fact that $\frac{\partial \tilde{M}_{T}}{\partial S_{t}}$ is in $L^{2}$.

Proposition 2.9. When the coefficients $r, \mu$ and $\sigma$ are Markov, and if we further impose that $F^{n}$ is twice continuously differentiable for all $n$, as well as (A1), (A2), (A3) and (A4) or (A'4), then the sequence $\left(\pi_{t}^{n}\right)$ converges almost surely to $\pi_{t}^{\infty}$ for all $t$. 


\section{References}

[1] Cox, J. C. and C.-F. Huang, 1989, Optimal consumption and portfolio policies when asset prices follow a diffusion process, Journal of Economic Theory, 49, 33-83.

[2] Cvitanic, J. and I. Karatzas, 1996, Hedging and portfolio optimization under transaction costs: A martingale approach, Mathematical Finance, 6, 133-165.

[3] Gihman, I. and A. Skorohod, 1972, "Stochastic differential equations", Springer-Verlag, New-York.

[4] Grasselli, M.; 2001, "Pension funds : a mathematical finance approach", PhD thesis, Université de Paris 1.

[5] Karatzas, I., 1989, Optimization problems in the theory of continuous trading, SIAM Journal of Control and Optimization, 27, 1221-1259.

[6] Karatzas, I., Lehoczky, J. P. and S.E. Shreve, 1987, Optimal portfolio and consumption decisions for a "small investor" on a finite horizon, SIAM Journal of Control and Optimization, 25, 1557-1586.

[7] Karatzas, I., Lehoczky, J. P., Shreve, S. E. and G. L. Xu, 1991, Martingale and duality methods for utility maximization in an incomplete market, SIAM Journal of Control and Optimization, 25, 1557-1586.

[8] Kramkov, D. and W. Schachermayer, 1999, The asymptotic elasticity of utility functions and optimal investment in incomplete market, Annals of Applied Probability, 9, 904 - 950.

[9] Merton, R. C., 1971, Optimum consumption and portfolio rules in a continuous-time model, Journal of Economic Theory, 3, 373-413.

[10] Rockafellar, R. T., 1970, "Convex analysis", Princeton University Press, Princeton, NewJersey. 